Highlights

Determinants of the primary stability of cementless acetabular cup implants: A 3D finite element study

Katharina Immel,Vu-Hieu Nguyen,Arnaud Dubory,Charles-Henri Flouzat-Lachaniette,Roger A. Sauer,Guillaume Haïat

- Polar gaps increase as a function of bone stiffness, interference fit, and friction

- Pull-out force increases continuously with increasing bone stiffness

- Pull-out force peaks for interference fit of $1.4 \mathrm{~mm}$ and friction coefficient of 0.6

- Optimal combinations of interference fit and friction maximize primary stability 


\title{
Determinants of the primary stability of cementless acetabular cup implants: A 3D finite element study
}

\author{
Katharina Immel $^{a, b}$, Vu-Hieu Nguyen ${ }^{c, d}$, Arnaud Dubory ${ }^{e, f}$, Charles-Henri Flouzat-Lachaniette ${ }^{e, f}$, \\ Roger A. Sauer ${ }^{a, g, h}$ and Guillaume Haïat ${ }^{b, *}$ \\ ${ }^{a}$ Aachen Institute for Advanced Study in Computational Engineering Science (AICES), RWTH Aachen University, Templergraben 55, 52056 Aachen, \\ Germany \\ ${ }^{b}$ MSME, CNRS UMR 8208, Université Paris-Est Créteil, Université Gustave Eiffel, 94010 Créteil, France \\ ${ }^{c}$ Université Paris-Est Créteil, CNRS, MSME, 94010 Créteil, France \\ ${ }^{d}$ Université Gustave Eiffel, MSME, 77454 Marne-la-Vallée, France \\ ${ }^{e}$ Service de Chirurgie Orthopédique et Traumatologique du Centre Hospitalier Universitaire Henri Mondor, 51 Avenue du Maréchal de Lattre de \\ Tassigny, 94000 Créteil, France \\ ${ }^{f}$ Équipe 10, Groupe 5, IMRB U955, INSERM/UPEC, 51 Avenue du Maréchal de Lattre de Tassigny, 94010 Créteil, France \\ ${ }^{g}$ Faculty of Civil and Environmental Engineering, Gdańsk University of Technology, ul. Narutowicza 11/12, 80-233 Gdańsk, Poland \\ ${ }^{h}$ Department of Mechanical Engineering, Indian Institute of Technology Kanpur, UP 208016, India
}

\section{ARTICLE INFO}

\section{Keywords:}

acetabular cup

finite element analysis

friction

interference fit

primary stability

osseointegration

\begin{abstract}
A B S TR ACT
Primary stability of cementless implants is crucial for the surgical success and long-term stability. However, primary stability is difficult to quantify in vivo and the biomechanical phenomena occurring during the press-fit insertion of an acetabular cup (AC) implant are still poorly understood. The aim of this study is to investigate the influence of the cortical and trabecular bone Young's moduli $E_{\mathrm{c}}$ and $E_{\mathrm{t}}$, the interference fit $I F$ and the sliding friction coefficient of the bone-implant interface $\mu$ on the primary stability of an AC implant. For each parameter combination, the insertion of the AC implant into the hip cavity and consequent pull-out are simulated with a 3D finite element model of a human hemi-pelvis. The primary stability is assessed by determining the polar gap and the maximum pullout force. The polar gap increases along with all considered parameters. The pull-out force shows a continuous increase with $E_{\mathrm{c}}$ and $E_{\mathrm{t}}$ and a non-linear variation as a function of $\mu$ and $I F$ is obtained. For $\mu>0.6$ and $I F>1.4$ the primary stability decreases, and a combination of smaller $\mu$ and $I F$ lead to a better fixation. Based on the patient's bone stiffness, optimal combinations of $\mu$ and $I F$ can be identified. The results are in good qualitative agreement with previous studies and provide a better understanding of the determinants of the AC implant primary stability. They suggest a guideline for the optimal choice of implant surface roughness and $I F$ based on the patient's bone quality.
\end{abstract}

\section{Introduction}

Total hip arthroplasty (THA) is one of the most common surgical procedures with a total of around 500,000 THA performed yearly in the United States alone [25]. However, surgical failures still occur and may have dramatic consequences, leading to revision surgery. Up to $25 \%$ of the patients have to undergo revision surgery, and approximately 7 $\%$ within the first 8 years of implantation [50], while the 15year survival rate for revision surgery is only $69 \%$. Aseptic loosening is one of the most common causes of failure [24] and is often determined by the implant primary stability [44].

Cementless acetabular cup implants (ACI) have become more and more common for THA surgery [48]. Initial stability is obtained during the surgical intervention through Haiat)

${ }^{*}$ Corresponding author. E-mail address: guillaume.haiat@cnrs.fr (G.

immel@aices.rwth-aachen.de (K. Immel); vu-hieu.nguyen@u-pec.fr (V. Nguyen); arnaud. dubory@aphp. fr (A. Dubory);

charles-henri flouzat-lachaniet te@aphp. fr (C. Flouzat-Lachaniette); sauer@aices. rwth-aachen. de (R.A. Sauer); guillaume. haiat@enrs. fr (G. Haïat)

ORCID(s): 0000-0001-7511-2910 (K. Immel); 0000-0003-1959-9087 (V. Nguyen); $0000-0001-7371-1645$ (A. Dubory); $0000-0003-3229-5758$ (C.

Flouzat-Lachaniette); 0000-0001-5625-8295 (R.A. Sauer);

0000-0003-1724-9083 (G. Haïat) a diameter interference fit, by reaming the host bone cavity $[23,28,29]$. The ACI is inserted into the bone cavity using impacts and the initial fixation is achieved through residual stresses, localized mostly at the ACI rim [17, 32]. Longterm stability is then obtained by bone growing around and into the porous surface of the implant [5], a process called osseointegration. To achieve an optimal primary stability, a compromise should be found between: 1 . reducing the relative micro-motions at the bone-implant interface (BII), 2. avoiding large gaps between bone and implant, which may lead to the formation of fibrous tissue in the peri-implant region [5], the formation of low-quality bone tissue or even inhibit bone growth $[22,32,41]$, and 3 . avoiding excessive stresses in peri-implant bone tissue, which may lead to bone necrosis or local ischemia [45]. All these phenomena may jeopardize osseointegration processes [22, 38, 42], and can lead to implant loosening. It remains difficult to predict ACI loosening because of its multi-factorial causes related to the implant properties, the cavity geometry (e.g., its diameter), and to the patient's bone quality $[1,18,26]$. Different pullout tests have been carried out in vitro and ex vivo to assess the ACI primary stability [13, 27], with several studies focusing on the effect of bone quality on the biomechanical behavior of the ACI $[18,21]$. However, such biomechani- 
cal testing cannot be applied during surgery and the aforementioned approaches may be related to empirical developments. It remains difficult to carry out a systematic in vitro investigation of the multifactorial determinants of ACI stability because of the difficulty to precisely control all parameters when using bone tissue.

For these reasons, several numerical models have been developed to assess the biomechanical behavior of the cementless ACI with simplified bone geometries [17, 38, 46, 54]. While such models offer some initial insight on the biomechanical parameters and the contact behavior of the BII in a controlled environment, their simplified geometry constitutes a strong limitation because it does not properly capture 3D effects that have an important influence on the structural behavior of the pelvis. In particular, Cilingir et al. (2007) [7] investigated the influence of the bone geometry on the contact pressure and stress distribution by comparing the performance of a 2D axisymmetric, 3D axisymmetric, and 3D hemi-pelvis model. They showed that, while all models predicted a similar contact pressure distribution in the acetabular cavity, the maximum von Mises stress within the bone tissue differed significantly. As the insertion of the ACI into the reamed cavity produces considerable stresses at the bone cavity rim, anatomic 3D models must be considered to achieve more reliable results. Consequently, more accurate models of the human pelvis have been developed to model the contact behavior of the ACI. These models can be subdivided into two categories: case-specific modeling [19] and multi-factorial studies $[1,2,18,21,40]$. A case-specific model is usually used to investigate a unique situation e.g., a specific condition or geometrical anomaly, whereas a multifactorial study aims to generate various cases representative of a population and may require a large number of analyses [8]. However, none of the aforementioned studies analyzed both the insertion and the stability of the ACI, which are highly interdependent. Moreover, only a small range of parameters were analyzed so far.

The aim of this work is to provide a better understanding of the determinants of the primary stability of cementless $\mathrm{ACI}$ in the human pelvis. The ACI primary stability is assessed through the estimation of the pull-out force [35] and the polar gap [1,29]. Therefore, geometrically nonlinear FE analyses were performed to simulate the quasi-static insertion and subsequent pull-out of the ACI in a patient's hemipelvis. The influence of a broad range of different implantand patient-specific parameters on the ACI primary stability, such as the friction coefficient at the BII $\mu$, the bone quality in terms of cortical and trabecular bone Young's moduli $E_{\mathrm{c}}$, $E_{\mathrm{t}}$, and the diametric interference fit $I F$, are analyzed and compared to a previous 2D study [38].

The remainder of this work is structured as follows: First, the setup of the FE model is described. Second, the results of the FE simulations and the parameter study are presented. The structural behavior of the hip during insertion and removal of the ACI, as well as the influence of every parameter on primary stability in terms of pull-out force and polar gap is discussed. For three different trabecular bone stiffnesses
$\left(E_{\mathrm{t}}=0.1,0.2,0.5 \mathrm{GPa}\right)$ optimal combination of values for $\mu$ and $I F$ are presented.

\section{Materials and Methods}

The choice of the input parameters and geometrical properties of the present FE model are based on the study of Raffa et al. (2019) [38]. In contrast to their work, the geometry of a real human pelvis was used here, instead of a simplified trabecular bone block. Finite element meshing and numerical analyses were carried out using ANSYS Workbench software (v.14, ANSYS, Inc., Canonsburg, PA, USA).

\subsection{Contact and material model}

Given two 3D bodies and their contact surfaces, the contact traction $t_{\mathrm{c}}$ can be decomposed into a normal and a tangential component [53], i.e.,

$$
t_{\mathrm{c}}=t_{\mathrm{n}}+t_{\mathrm{t}} .
$$

The normal traction can be expressed as

$$
\boldsymbol{t}_{\mathrm{n}}=p \boldsymbol{n},
$$

where $p$ is the contact pressure and $\mathrm{n}$ is the surface outward normal. For frictional contact, the tangential traction is determined by the behavior during sticking and sliding, and the distinction between these two cases is based on a slip criterion of the form

$$
f_{\mathrm{s}} \begin{cases}<0, & \text { for sticking, } \\ =0, & \text { for sliding. }\end{cases}
$$

In this work, frictional contact is modeled by Coulomb's law for which Eq. (3) can be formulated as

$$
f_{\mathrm{s}}=\left(\left\|t_{\mathrm{t}}\right\|-\mu p\right) \leq 0,
$$

with constant friction coefficient $\mu$. The normal reaction force may become positive to compensate the friction force occurring at the BII when pulling the implant out of the cavity. Throughout this work, an isotropic, homogeneous material model is used for all bodies. For large deformation, ANSYS uses the hypoelastic material model

$$
\check{\boldsymbol{\sigma}}=\frac{E v}{(1+v)(1-2 v)} \operatorname{tr}(\boldsymbol{D}) \boldsymbol{I}+\frac{E}{(1+v)} \boldsymbol{D},
$$

based on the Jaumann rate of the Cauchy stress

$$
\check{\boldsymbol{\sigma}}=\dot{\boldsymbol{\sigma}}+\boldsymbol{\sigma} \cdot \boldsymbol{w}-\boldsymbol{w} \cdot \boldsymbol{\sigma},
$$

where $E$ is the Young's modulus, $v$ is the Poisson ratio, and $\boldsymbol{I}$ is the identity matrix. The rate of deformation tensor $\boldsymbol{D}$ and the spatial spin tensor $\boldsymbol{w}$ are derived from the velocity $\boldsymbol{v}$ as

$$
\boldsymbol{D}=\frac{1}{2}\left(\nabla \boldsymbol{v}+\nabla \boldsymbol{v}^{\mathrm{T}}\right), \quad \boldsymbol{w}=\frac{1}{2}\left(\nabla \boldsymbol{v}-\nabla \boldsymbol{v}^{\mathrm{T}}\right) .
$$




\subsection{Geometry and mesh}

The geometry for the human hemi-pelvis was obtained from a free online data base [15]. The points comprising the contours of the hip were triangulated, decimated, and smoothed in MeshLab [6]. The thickness of the cortical bone layer may vary and its limit with the trabecular bone is blurred because the properties of the bone change gradually, which is not taken into account in our model. Instead, we assumed a uniform thickness of the cortical bone tissue of $1 \mathrm{~mm}$ [47]. The corresponding cortical layer was created by extrusion with Meshmixer (Autodesk, San Rafael, CA, USA). A hemi-spherical cavity was created in the acetabula, using ANSYS Workbench. The cavity diameter was varied between 48.5 and $50.9 \mathrm{~mm}$, which corresponds to different values of the diametric interference fit $I F(0-2.5 \mathrm{~mm})$, according to values found in the literature [17, 26, 28, 47, 54]. The resulting pelvis geometry is shown in Figure $1 \mathrm{~A}$ and $\mathrm{B}$.

The ACI was modeled after the Cotyle Cerafit (Ceraver, Roissy, France) and has an outer radius of $25.5 \mathrm{~mm}$. Its thickness varies linearly as a function of the polar angle, with values between $2.9 \mathrm{~mm}$ at the cup dome and $3.7 \mathrm{~mm}$ at the cup rim, similarly as what is used in previous studies [30, 38]. Moreover, a cylindrical ancillary, with a radius and height of $8.5 \mathrm{~mm}$ and $190 \mathrm{~mm}$, was rigidly attached to the ACI, as it is done in the clinic during insertion. The complete model is shown in Figure 1A.

The FE mesh was generated with ANSYS Workbench and it slightly varies for every value of the interference fit IF . It typically contains approximately 125,000 tetrahedral and hexahedral, quadratic Lagrangian elements, leading to a global system with approximately 615,000 degrees of freedom. The mesh size is finer around the bone cavity surface (average size $0.8 \mathrm{~mm}$ ) to provide a sufficiently accurate geometrical approximation of the curved contact surface. An enlargement of the mesh at the cavity is shown in Figure 1B. A standard convergence study concerning the element size $h_{e}$ and the load step increment $\Delta l s$ was performed for the reference case, with the pull-out force as the convergence criteria. The mesh and load step increment were refined until the relative change in the pull-out force was below $1 \%$ and quadratic Newton-Raphson convergence within 2 steps was ensured. In cases where parameter combinations with high stiffness or interference fit did not converge for the determined load step increment, the load step increment was further decreased.

\subsection{Material properties and varied parameters}

The ancillary and the ACI were assumed to be made of stainless steel and titanium alloy (Ti-Al6-V4), respectively. The pelvis was assumed to be composed of a uniform thin outer layer of cortical bone $(1 \mathrm{~mm})$ and trabecular bone inside. However, due to the simulated reaming, no cortical bone remained on the contact surface of the hip cavity (see Figure 1B). All materials were assumed to be homogeneous, isotropic, and hypoelastic. The Poisson ratio for all materials was assumed to be $v=0.3$. Table 1 shows the elastic properties of the different materials used in this study. Fric- tion was modeled with a standard Coulomb's law (4), with constant friction coefficient $\mu$. A wide range of values of $\mu$ (between 0 and 1) was considered in order to simulate the physiological range of friction for various types of implant surfaces in contact with bone and for different clinical situations of the BII $[11,34,47] . \mu^{*}=0.3$ was taken as the reference value [11]. Variations of the Young's moduli of cortical bone $E_{\mathrm{c}}$ and trabecular bone $E_{\mathrm{t}}$ were considered within their physiological range [21, 37, 43, 51] and for some extreme cases: $E_{\mathrm{t}}$ between 0.1 and $2 \mathrm{GPa}$ and $E_{\mathrm{c}}$ between 0.2 and $25 \mathrm{GPa}$. The bone's elastic modulus in the cavity provides an indication of the bone quality and has been previously investigated in FE studies [18, 21].

Optimal parameter combinations of $I F$ and $\mu$ for three different trabecular bone stiffness $E_{\mathrm{t}}=0.1,0.2,0.5 \mathrm{GPa}$ were determined. The reference values for $E_{\mathrm{c}}^{*}=18 \mathrm{GPa}$ [43] and $E_{\mathrm{t}}^{*}=0.2 \mathrm{GPa}$ [37] were chosen according to the literature. A broad range of the diametric interference fit IF (between 0 and $2.5 \mathrm{~mm}$ ) was considered, to cover most commonly used configurations, as well as extreme cases. The corresponding reference value was assumed to be $I F^{*}=1$ $\mathrm{mm}$, which is a standard value used in clinical practice [26]. Throughout this study, the parameter set of $E_{\mathrm{c}}^{*}=18 \mathrm{GPa}$, $E_{\mathrm{t}}^{*}=0.2 \mathrm{GPa}, \mu^{*}=0.3$, and $I F^{*}=1.0 \mathrm{~mm}$ is referred to as the reference case. Table 1 lists the analyzed parameters with their corresponding range and reference value.

\subsection{Boundary and loading conditions and solver settings}

All simulations were performed with quasi-static analysis setting (i.e., excluding inertia and viscosity) and large deformation effects. Frictional contact with the augmented Lagrange method was used. The hip cavity was set as the slave surface and the ACI was set as the master surface.

The pelvis was rigidly fixed in all directions at the pubic symphysis and the iliac joint (see Figure 1A, red), following the literature $[2,8,16,43]$. All other location corresponding to bone tissue were free. At the initial state (load step) $l s_{0}=$ 0 , the outer boundary of the ACI and the internal boundary of the hip cavity were assumed to be close but not in contact.

To simulate the insertion process, a uniform displacement $d_{0}$ was applied to the ancillary. The implant was displaced by $d_{0}$ until the normal reaction force reached $F_{0}=$ $-2500 \mathrm{~N}$. The reaction force $F_{0}$ was chosen based on experimental measurements from Raffa et al. (2019) [38] and is similar to values found in the literature [27, 45, 46]. The displacement $d_{0}$ depends on the studied parameters and is not known a priori. It was computed for each parameter combination individually by performing an initial insertion simulation first to determine $d_{0}\left(\mu, I F, E_{\mathrm{c}}, E_{\mathrm{t}}, F_{0}\right)$. To simulate the push-in and removal of the implant into and from the pelvis, the simulation was divided into three stages: First, the displacement $d_{0}$ was applied to the top surface of the ancillary (see Figure 1A, blue) in order to insert the implant into the hip until the normal reaction force $F_{0}$ was reached at load step $l s_{1}=200$. Second, the implant and the ancillary were held in place until load step $l s_{2}=240$. This was done only 

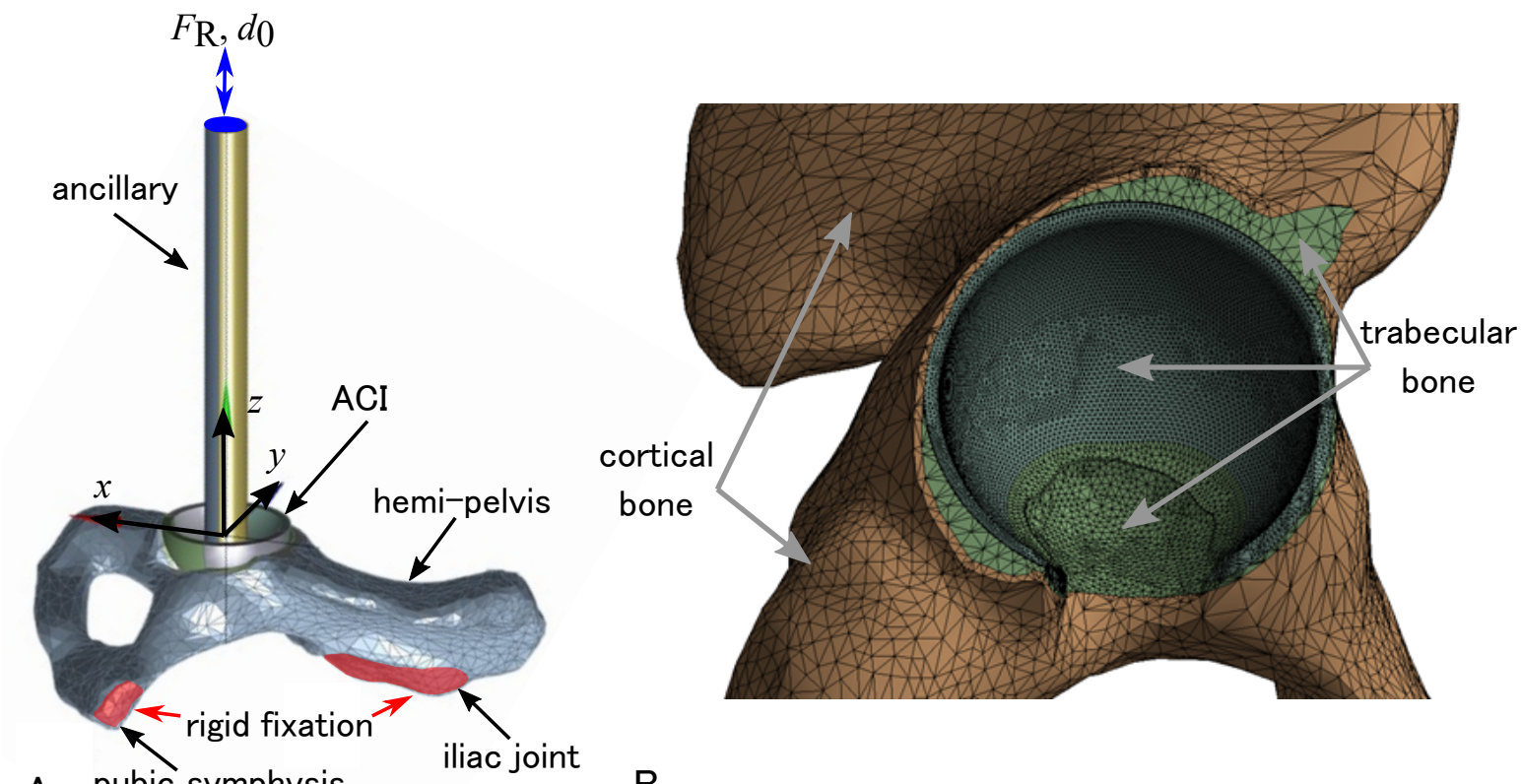

A pubic symphysis

Figure 1: (A) Image of the pelvis geometry with the $\mathrm{ACl}$ and ancillary. Red elements indicate fixed rigid boundary conditions. Blue elements indicate the region where the displacement is applied. (B) Enlargement of the FE mesh at the cavity. Brown elements indicate cortical bone and green elements represent trabecular bone.

Table 1

Material properties of the four subdomains as well as ranges and reference values of the studied parameters.

\begin{tabular}{lllll}
\hline Domain/Parameter & Material & Symbol & Range & Reference value \\
\hline Ancillary & Stainless steel & - & - & $210 \mathrm{GPa}$ \\
Implant & Ti-Al6-V4 & - & - & $113 \mathrm{GPa}$ \\
Outer bone & Cortical bone & $E_{\mathrm{c}}$ & $0.2-25 \mathrm{GPa}[43,51]$ & $18 \mathrm{GPa}[43]$ \\
Inner bone & Trabecular Bone & $E_{\mathrm{t}}$ & $0.1-2 \mathrm{GPa}[21,37]$ & $0.2 \mathrm{GPa}[37]$ \\
Interference fit & - & $I F$ & $0-2.5 \mathrm{~mm}[26,28,47]$ & $1 \mathrm{~mm}[26]$ \\
Friction coefficient & - & $\mu$ & $0-1[11,34,47]$ & $0.3[11]$ \\
\hline
\end{tabular}

for illustrative purposes; the actual holding time has no influence in quasi-static simulations. Third, the uniform pull-out displacement $-d_{0}$ was applied to the top surface of the ancillary, until the implant is completely detached from the bone (load step $l s_{3}=440$ ). The quasi-static displacement uz in axial direction $\mathrm{z}$ can then be described by

$$
u_{z}= \begin{cases}d_{0} \cdot \Delta l s / s_{1} & \text { for } l s_{0} \leq l s<l s_{1} \\ d_{0} & \text { for } l s_{1} \leq l s<l s_{2} \\ d_{0} \cdot\left(l_{3}-\Delta l s\right) / s_{1} & \text { for } l s_{2} \leq l s \leq l s_{3}\end{cases}
$$

In most cases the load step size of $\Delta l s=d_{0} / 200$ was used during the insertion and the extraction phases (resulting in 440 load steps). For high friction coefficients (0.6-1.0) and a critical interference fit (between 1.0 and $1.8 \mathrm{~mm}$ ), a smaller load step size, such as $\Delta l s=d_{0} / 5000$ was needed to ensure Newton-Raphson convergence.

\subsection{Quantifying primary stability}

In this work, the primary stability is quantified by the size of the polar gap after insertion and by the values of the pull-out force. The pull-out force $F_{\mathrm{p}}$ is defined by the positive maximum normal reaction force $F_{\mathrm{R}}$ obtained at the upper surface of the ancillary during the pull-out phase. The polar gap is determined as the distance between the pole of the ACI and the pole of the hip cavity during the holding phase (load steps $l s=200-240$ ). The pull-out force has already been used in previous works in order to assess the ACI primary stability $[4,27,35,38,47]$. The pull-out force is a clinically relevant quantity because after inserting the ACI in the pelvis, surgeons usually attempt to pull or lever-out the ACI to check manually for the stability and motion of the ACI. Furthermore, it is commonly used as a determinant of primary stability in in vitro studies [13, 18, 21, 27]. Moreover, the polar gap between the ACI and the hip is also frequently used in clinical studies $[21,30,40]$ in order to assess the ACI stability. Large gaps indicate improper seating of the ACI and can affect the quality of long-term fixation due to the formation of fibrous tissue in the peri-implant region [5], the formation of low-quality bone tissue or even inhibit bone growth [22, 32, 41]. The polar gap is evaluated based on values found in the literature [22, 29, 42, 49], where 
gaps below $500 \mu \mathrm{m}$ (indicated in green in Figures 4B-7B) are considered optimal, gaps between $500 \mu \mathrm{m}$ and $1 \mathrm{~mm}$ are considered acceptable (indicated in yellow), and gaps above $1 \mathrm{~mm}$ (indicated in red) are considered to be critical.

\section{Results}

The results obtained with the FE model are presented and compared to a previous 2D study [38]. First, the main differences between the results obtained in the previous $2 \mathrm{D}$ axisymmetric study and in the present study for the reference case are discussed. Second, the structural response of the pelvis and the implant in terms of stress distribution is analyzed. Last, the parametric study on the influence of the bone Young's moduli $E_{\mathrm{t}}$ and $E_{\mathrm{c}}$, the friction coefficient $\mu$, and the interference fit $I F$ on primary stability as well as optimal combinations of these parameters are discussed.

\subsection{Reference case}

Figure 2 shows the variation of the vertical reaction force $F_{\mathrm{R}}$ at the upper surface of the ancillary as a function of time for the reference case. The results are compared with those obtained in the 2D axisymmetric study of Raffa et al. (2019) [38]. Three phases of the controlled displacement can be identified:

1. an insertion phase from $l s_{0}=0$ to $l_{1}=200$. As soon as contact is established, the reaction force decreases as a function of the displacement until the predefined value $F_{0}=-2500 \mathrm{~N}$ is reached. During the insertion phase, the slope of $F_{\mathrm{R}}$ is first constant, which corresponds to constant stiffness of the bone-implant system.

2. a holding phase from $l_{1}=201$ to $l_{2}=240$, where the implant is held in place. Here, the reaction force is constant with $F_{\mathrm{R}}=F_{0}$.

3. a pull-out phase from $l_{2}=241$ to $l s_{3}=440$, until the ACI is completely removed, and no contact remains. The reaction force increases up to a positive maximum $F_{\mathrm{p}}$, and then decreases to 0 .

Figure 3 shows the distribution of the von Mises stress $\sigma_{\mathrm{M}}$ in the hip cavity at $l s=220$ during the holding phase. The maximal von Mises equivalent stress of $407 \mathrm{MPa}$ is reached after full insertion and is localized in several elements of the cortical shell around the acetabulum. The von Mises equivalent stress inside the trabecular bone of the cavity is significantly lower than in the cortical shell. Inside the contact area, the highest stresses are found in the equatorial rim with approximately $10 \mathrm{MPa}$. The stress distribution at the time where the maximal pull-out force is reached remains similar to the holding phase. However, the maximum stress only reaches $267 \mathrm{MPa}$ during pull-out.

\subsection{Effect of variations of the trabecular bone Young's modulus $E_{\mathrm{t}}$}

Figure 4 shows the variation of the pull-out force $F_{\mathrm{p}}(\mathrm{A})$ and the polar gap (B) as a function of the trabecular bone
Young's modulus $E_{\mathrm{t}}$ for the 2D and the 3D model. While the results of the 2D model show a distinct peak of the pull-out force at $F_{\mathrm{p}}=667 \mathrm{~N}$ for $E_{\mathrm{t}} \approx 0.375 \mathrm{GPa}$, the 3D hemi-pelvis model behaves differently since the pull-out force is an increasing function of $E_{\mathrm{t}}$. A similar behavior can be observed for the polar gap (B), which increases as a function of $E_{\mathrm{t}}$. Note that the variation of the polar gap was not investigated in the $2 \mathrm{D}$ case, so no comparison is possible with the $3 \mathrm{D}$ case.

\subsection{Effect of variations of the cortical bone Young's modulus $E_{\mathrm{c}}$}

Figure 5 shows the variation of the pull-out force $F_{\mathrm{p}}$ (A) and the polar gap (B) as a function of the cortical bone Young's modulus $E_{\mathrm{c}}$ for the present $3 \mathrm{D}$ model. A variation of the Young's modulus of cortical bone $E_{\mathrm{c}}$ within the physiological range $(15-23 \mathrm{GPa}[43,51])$ only has a negligible effect on the pull-out force (207-219 N). The pull-out force only decreases for extreme cases with very low cortical bone Young's modulus ( $<10 \mathrm{GPa})$. Up to $E_{\mathrm{c}}=8 \mathrm{GPa}$, the polar gap increases only slightly and is less than several micrometers and may therefore be considered as negligible. In contrast to $E_{\mathrm{t}}$, all tested values of $E_{\mathrm{c}}$ result in acceptable polar gaps $(<500 \mu \mathrm{m})$. Therefore, the influence of $E_{\mathrm{c}}$ within its physiological range is deemed negligible and only different values of $E_{\mathrm{t}}$ will be considered in what follows.

\subsection{Effect of variations of the friction coefficient $\mu$}

Figure 6 shows the variation of the pull-out force $F_{\mathrm{p}}(\mathrm{A})$ and the polar gap (B) as a function of the friction coefficient $\mu$ for different values of $E_{\mathrm{t}}$. The results obtained here are compared with the 2D case. $F_{\mathrm{p}}$ increases for low values of $\mu$, reaches a maximum at around $\mu=0.6$, and then slowly decreases for all models and values of $E_{\mathrm{t}}$. The pull-out forces obtained with the present $3 \mathrm{D}$ model are approximately halved, compared to the results of the 2D study. For $\mu<0.17$ the pull-out force is zero for all values of $E_{\mathrm{t}} . F_{\mathrm{p}}$ increases as a function of the trabecular bone stiffness, while maintaining a similar behavior for various friction coefficients. The polar gap increases almost linearly as a function of the friction coefficient when a constant insertion force is considered, which can be explained by the fact that a high value of the friction coefficient inhibits the insertion process. Increasing the value of $\mu$ from 0.3 to 0.6 leads to an increase of the polar gap from $162 \mu \mathrm{m}$ and $614 \mu \mathrm{m}$, which is above the maximum recommended gap of $500 \mu \mathrm{m}[9,42]$. With a friction coefficient of $0.5, F_{\mathrm{p}}=476 \mathrm{~N}$ and the polar gap is equal to $455 \mu \mathrm{m}$. In the present case, all values of $\mu>0.5$ lead to polar gaps higher than $500 \mu \mathrm{m}$ and values of $\mu>0.8$ lead to values of the polar gap exceeding the commonly reported limit of $1 \mathrm{~mm}[22,29]$. For $E_{\mathrm{t}}=0.1 \mathrm{GPa}$, polar gaps below $500 \mu \mathrm{m}$ are achieved with $\mu<0.9$, while for $E_{\mathrm{t}}=0.5 \mathrm{GPa}$ only $\mu<0.3$ results in sufficiently small polar gaps when an interference fit of $I F=1 \mathrm{~mm}$ is considered.

\subsection{Effect of variations of the interference fit $I F$}

Figure 7 shows the variation of the pull-out force $F_{\mathrm{p}}$ (A) and the polar gap (B) as a function of the interference 


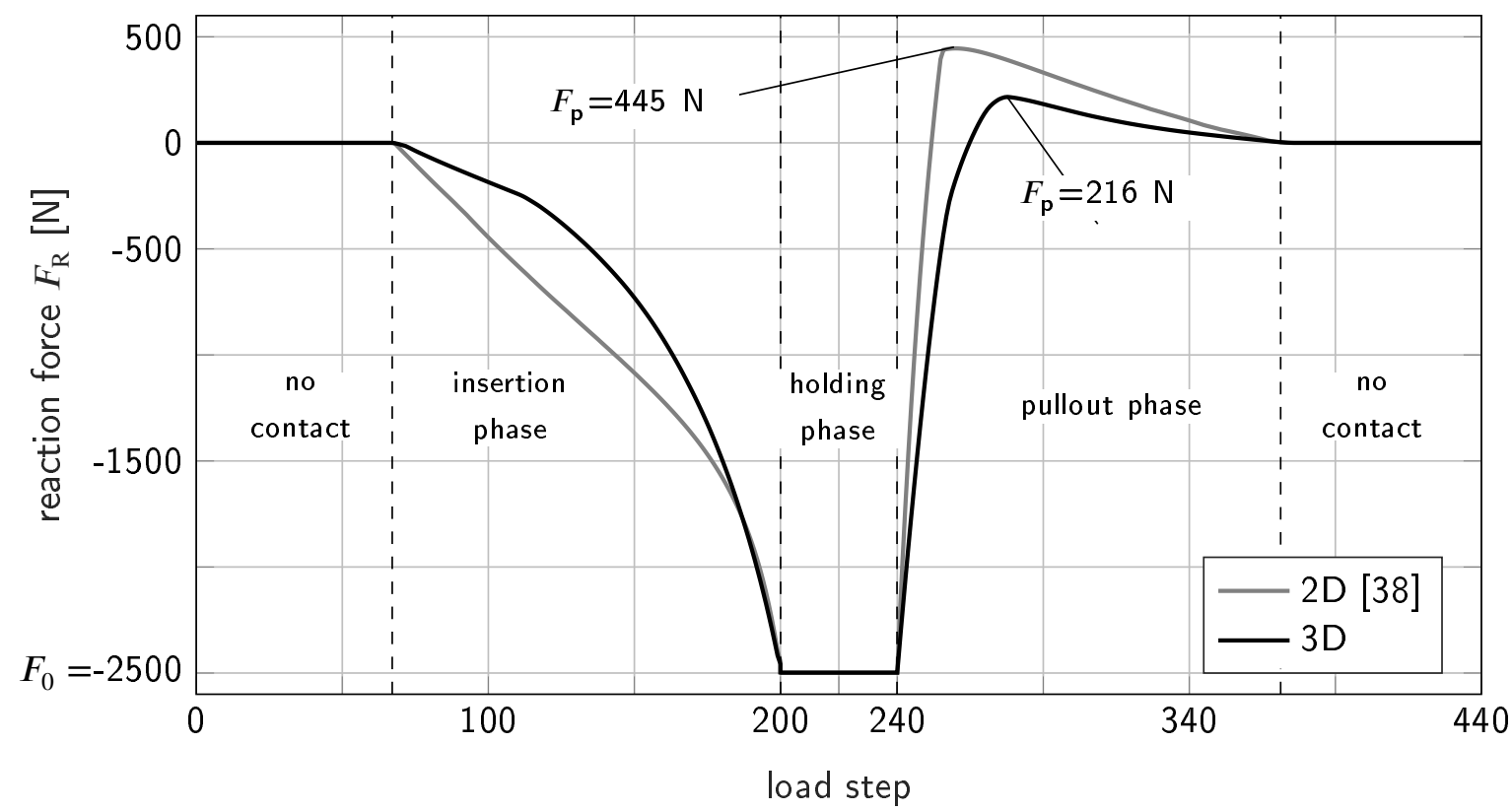

Figure 2: Variation of the reaction force $F_{\mathrm{R}}$ applied to the ancillary as a function of the load step $l s$ for the $2 \mathrm{D}$ and $3 \mathrm{D}$ model for the reference case.

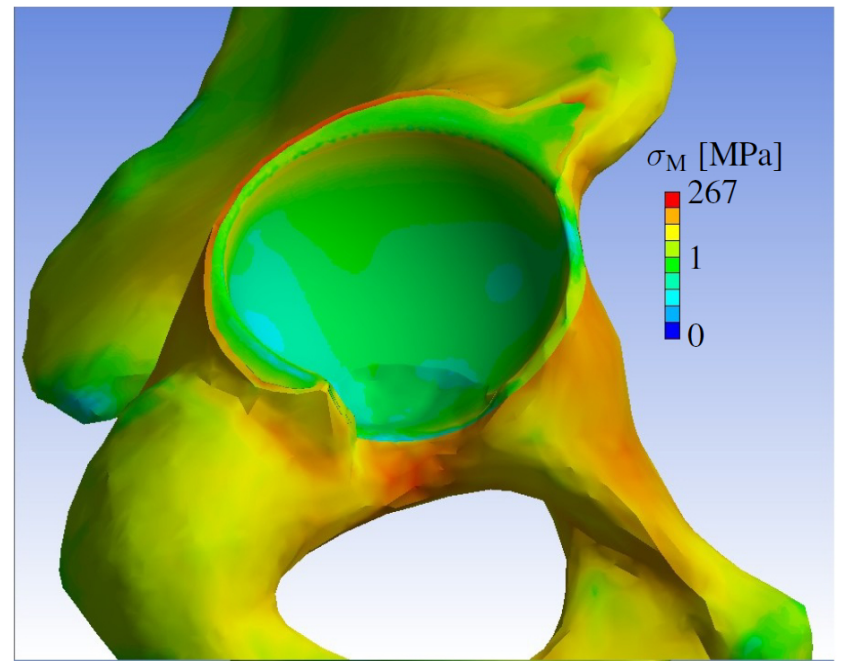

Figure 3: Distribution of the von Mises stress $\sigma_{\mathrm{M}}$ in the hip cavity for the reference case after insertion $(l s=220)$.

fit $I F$ for different values of $E_{\mathrm{t}}$. The results obtained with the model presented here are compared with the 2D case (in gray). A non-linear behavior of $F_{\mathrm{p}}$ as a function of $I F$ is obtained, similarly to the behavior of $F_{\mathrm{p}}$ as a function of $\mu$. For both, the 2D axisymmetric model and the 3D geometry, $F_{\mathrm{p}}$ reaches a maximum value for an optimal interference fit of $I F=1.2-1.4 \mathrm{~mm}$ for $E_{\mathrm{t}}^{*}=0.2 \mathrm{GPa}$. The pull-out force behaves similarly for different bone stiffness with a maximum value of $174 \mathrm{~N}$ for $E_{\mathrm{t}}=0.1 \mathrm{GPa}$ and $361 \mathrm{~N}$ for $E_{\mathrm{t}}=0.5 \mathrm{GPa}$. Moreover, the polar gap increases as a function of the interference fit for all values of $E_{\mathrm{t}}$. A polar gap equal to $162 \mu \mathrm{m}$ is obtained for the reference case and for $I F=1.4 \mathrm{~mm}$, the polar gap is equal to $493 \mu \mathrm{m}$. In the reference case, IF val- ues above $1.4 \mathrm{~mm}$ lead to polar gaps values higher than 500 $\mu \mathrm{m}$ and $I F$ values above $1.8 \mathrm{~mm}$ lead to polar gaps higher than $1 \mathrm{~mm}$. For $E_{\mathrm{t}}=0.1 \mathrm{GPa}$, acceptable gaps are achieved with $I F<2 \mathrm{~mm}$, and for $E_{\mathrm{t}}=0.5 \mathrm{GPa}$ with $I F<1 \mathrm{~mm}$.

\subsection{Optimal values of the friction coefficient and of the interference fit}

In what follows, the optimal combinations of $\mu$ and $I F$ for primary stability of the ACI are discussed. Values or parameter sets that maximize the pull-out force while maintaining a polar gap of lower than $500 \mu \mathrm{m}$ for a given bone stiffness are denoted optimal. Figure 8 shows the variation of the pull-out force as a function of the interference fit $I F$ and the friction coefficient $\mu$ for $E_{\mathrm{t}}^{*}=0.2 \mathrm{GPa}$ for the 3D model. The optimal interference fit $I F_{\text {opt }}$ for each $\mu$ is marked with a diamond. The variation of the maximum pull-out force $F_{\mathrm{p}}$ obtained for $I F_{\text {opt }}$ as a function of $\mu$ is shown in Figure 10 and the variation of the optimal interference fit $I F_{\text {opt }}$ as a function of $\mu$ is shown in Figure 9. The lowest value obtained for the optimal $I F$ is $I F_{\text {opt }}=0.2$ for $\mu=0.15$ and the highest is $I F_{\text {opt }}=1.4 \mathrm{~mm}$ for $\mu^{*}=0.3$ in the $3 \mathrm{D}$ model. The variation of the optimal interference fit $I F_{\text {opt }}$ as a function of $\mu$ is qualitatively similar in the 2D and the $3 \mathrm{D}$ case. However, the maximum pull-out force obtained with the determined $I F_{\text {opt }}$ for the 3D case are consistently lower than in the $2 \mathrm{D}$ case. Figure 10 shows that increasing $\mu$ higher than 0.6 does not lead to a higher pull-out force, which is consistent with the results shown in Figure 7A. Similar computations have also been done for $E_{\mathrm{t}}=0.1$ and $0.5 \mathrm{GPa}$ (data not shown) and the results show that an optimal case is also reached between $\mu_{\text {opt }}=0.5-0.6$. Overall, the optimal parameter sets with respect to the three values of trabecular bone stiffness $E_{\mathrm{t}}$ are shown in Table 2 . 


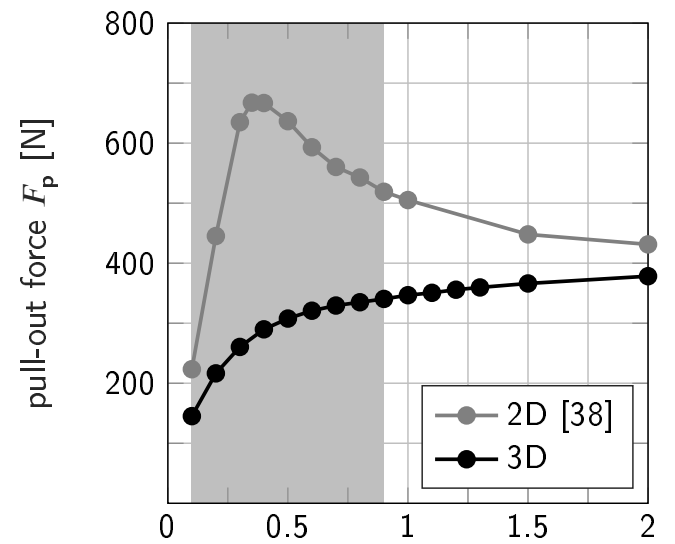

A

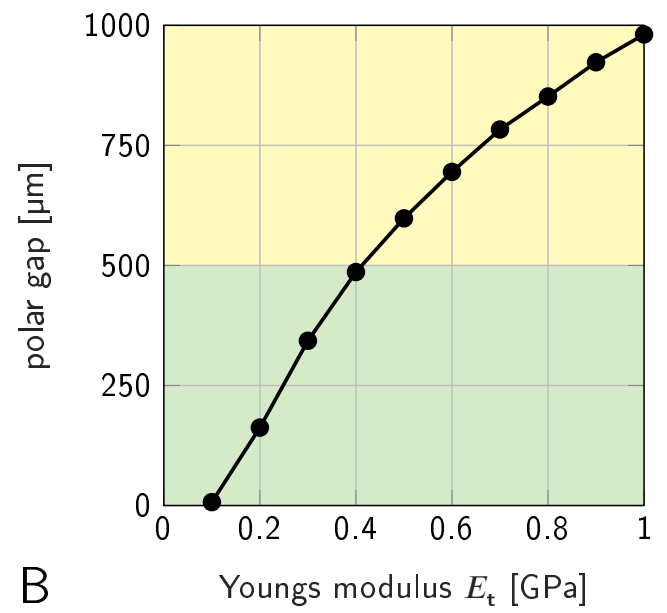

Figure 4: Variation of $(A)$ the pull-out force $F_{p}$ and $(B)$ the polar gap as a function of the Young's modulus of cortical bone $E_{\mathrm{c}}$ for constant trabecular Young's modulus $E_{\mathrm{t}}^{*}=0.2$ $\mathrm{GPa}$, friction coefficient $\mu^{*}=0.3$, and interference fit $I F^{*}=$ $1 \mathrm{~mm}$. The physiological range for $E_{\mathrm{t}}[21,37]$ is indicated in gray. The polar gap is classified as optimal $(<500 \mu \mathrm{m}$, green), acceptable ( $<1 \mathrm{~mm}$, yellow), or critical $(>1 \mathrm{~mm}$, red) $[22,29]$.

\section{Table 2}

Optimal values of the interference fit $I F_{\text {opt }}$ and the friction coefficient $\mu_{\text {opt }}$ with respect to trabecular bone stiffness $E_{\mathrm{t}}$.

\begin{tabular}{lllll}
\hline$E_{\mathrm{t}}[\mathrm{GPa}]$ & $I F_{\text {opt }}[\mathrm{mm}]$ & $\mu_{\text {opt }}$ & $F_{\mathrm{p}}[\mathrm{N}]$ & polar gap $[\mu \mathrm{m}]$ \\
\hline 0.1 & 0.8 & 0.6 & 304 & 190 \\
0.2 & 0.8 & 0.6 & 426 & 304 \\
0.5 & 0.8 & 0.6 & 569 & 475 \\
\hline
\end{tabular}

\section{Discussion}

This work aims to provide more insight into the biomechanical determinants of the primary stability of an ACI, which is estimated through the assessment of the pull-out force and the polar gap. The ACI primary stability is shown to depend on the elastic properties of the different bone tissues $\left(E_{\mathrm{t}}\right.$ and $\left.E_{\mathrm{c}}\right)$, on the friction coefficient $\mu$, and on the
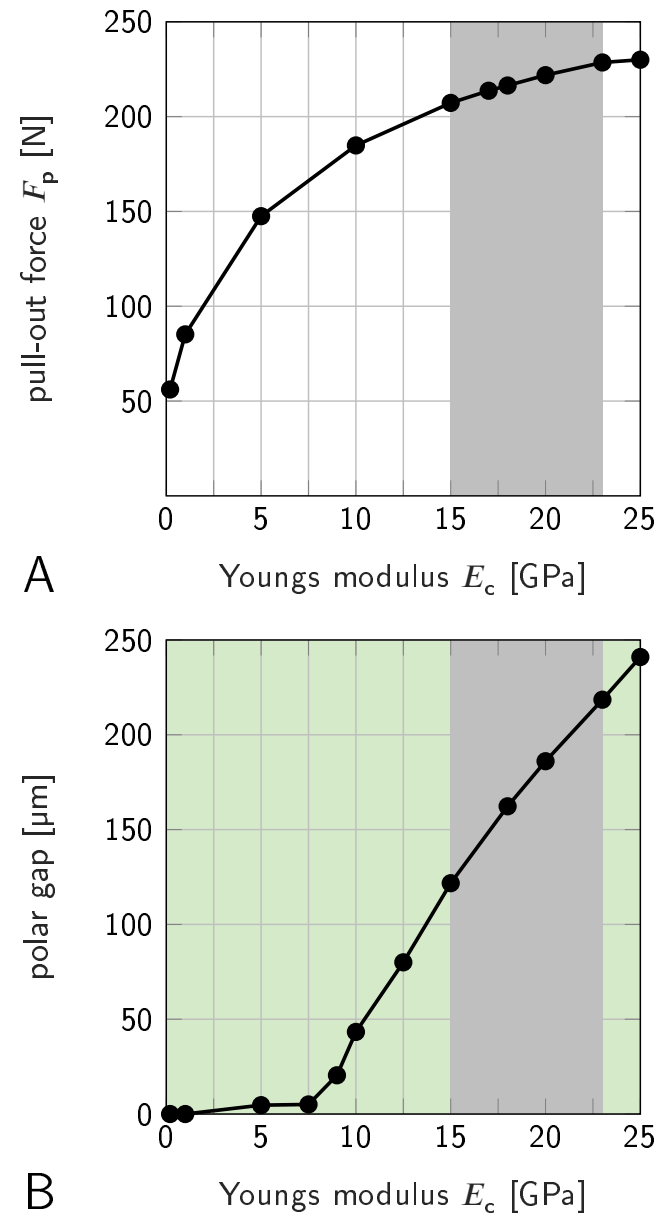

Figure 5: Variation of (A) the pull-out force $F_{\mathrm{p}}$ and $(\mathrm{B})$ the polar gap as a function of the Young's modulus of cortical bone $E_{\mathrm{c}}$ for constant trabecular Young's modulus $E_{\mathrm{t}}^{*}=0.2$ $\mathrm{GPa}$, friction coefficient $\mu^{*}=0.3$, and interference fit $I F^{*}=$ $1 \mathrm{~mm}$. The physiological range for $E_{\mathrm{c}}[43,51]$ is indicated in gray. The polar gap is classified as optimal $(<500 \mu \mathrm{m}$, green), acceptable ( $<1 \mathrm{~mm}$, yellow), or critical $(>1 \mathrm{~mm}$, red) $[22,29]$.

interference fit $I F$. The influence of these four parameters on the ACI primary stability was investigated within their respective physiological range (see Table 1). For each parameter set, the insertion of an ACI into a hip is simulated using a realistic FE model. When considering variations within their physiological ranges, $E_{\mathrm{t}}$ is shown to have a stronger influence on the pull-out force and the polar gap than $E_{\mathrm{c}}$, which can be explained by the fact that no cortical bone is present in the cavity. The results show that a maximum value of the pull-out force is obtained for specific combinations of $\mu$ and $I F$ (which depend on $E_{\mathrm{t}}$ ), while the polar gap increases as a function of all parameters. Based on these findings, optimal conditions for different bone stiffness can be determined and related to $\mu$ and to $I F$.

\subsection{Pull-out force}

The pull-out forces are found to be between 0 and $496 \mathrm{~N}$ in this study, which agrees with the results found in the lit- 
Determinants of primary stability of $\mathrm{ACl}$
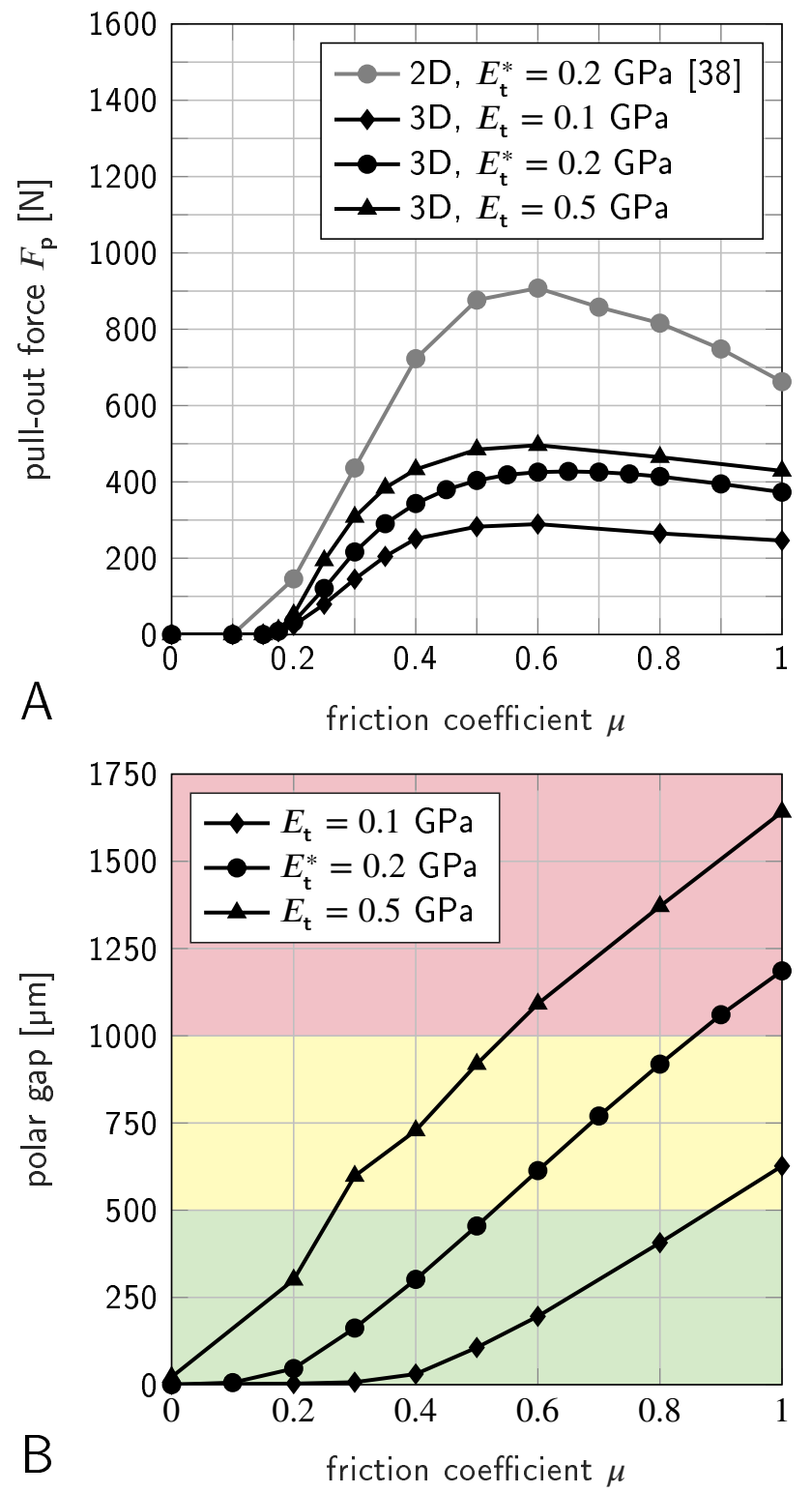

Figure 6: Variation of $(A)$ the pull-out force $F_{\mathrm{p}}(2 \mathrm{D}$ and $3 \mathrm{D}$ model) and ( $B$ ) the polar gap (3D only) as a function of the friction coefficient $\mu$ and trabecular Young's modulus $E_{\mathrm{t}}$. The results are shown for constant cortical Young's modulus $E_{c}^{*}=$ $18 \mathrm{GPa}$ and interference fit $I F^{*}=1 \mathrm{~mm}$. The polar gap is classified as optimal ( $<500 \mu \mathrm{m}$, green), acceptable $(<1 \mathrm{~mm}$, yellow), or critical (>1 mm, red) [22, 29].

erature [27, 38, 47]. The pull-out force increases along with the friction coefficient up to $\mu=0.6$, which can be explained by an increase of the tangential contact stresses at the equatorial rim, and then decreases because higher values of the friction coefficient inhibit sufficient seating of the cup (see Figure 7A) and thus removal becomes easier [47]. From the point of view of implant manufacturers, the results shown in Figure 7A indicate that increasing the friction coefficient beyond 0.6 may weaken the ACI primary stability. However, rough surfaces may enhance osseointegration phenomena [9] and thus long-term stability, which is another con-
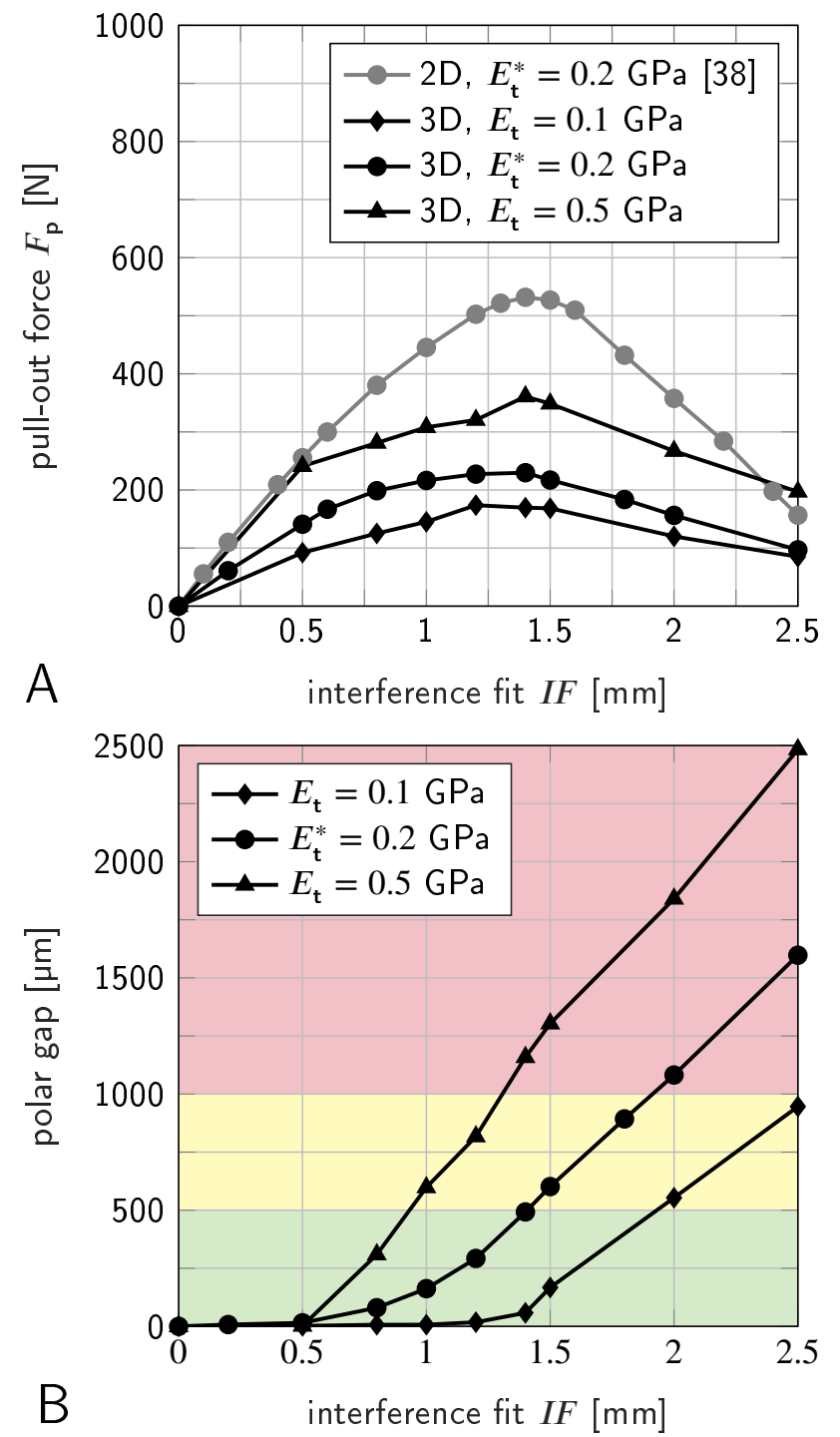

Figure 7: Variation of the pull-out force $F_{\mathrm{p}}$ (2D and 3D model) and the polar gap (3D only) as a function of the interference fit IF and trabecular Young's modulus $E_{\mathrm{t}}$. The results are shown for constant cortical bone Young's modulus $E_{c}^{*}=18 \mathrm{GPa}$, and friction coefficient $\mu^{*}=0.3$. The polar gap is classified as optimal ( $<500 \mu \mathrm{m}$, green), acceptable ( $<1 \mathrm{~mm}$, yellow), or critical ( $>1 \mathrm{~mm}$, red) $[22,29]$.

straint that should be taken into account. It has been shown that the pull-out force cannot be taken as the sole determinant of implant stability, as a parameter set with a maximal pull-out force can lead to excessive values of the polar gap, which can inhibit bone ingrowth and thus long-term implant stability [32, 41].

The pull-out forces predicted in the present study are significantly lower than the ones found in comparable 2D axisymmetric studies [38, 47]. Moreover, while the results of the 2D model showed that the pull-out force reaches a maximum value of $F_{\mathrm{p}}=667 \mathrm{~N}$ for $E_{\mathrm{t}} \approx 0.375 \mathrm{GPa}$, the behavior of the pull-out force predicted by the 3D hemipelvis model is different (see Figure 4A) since the pull-out 


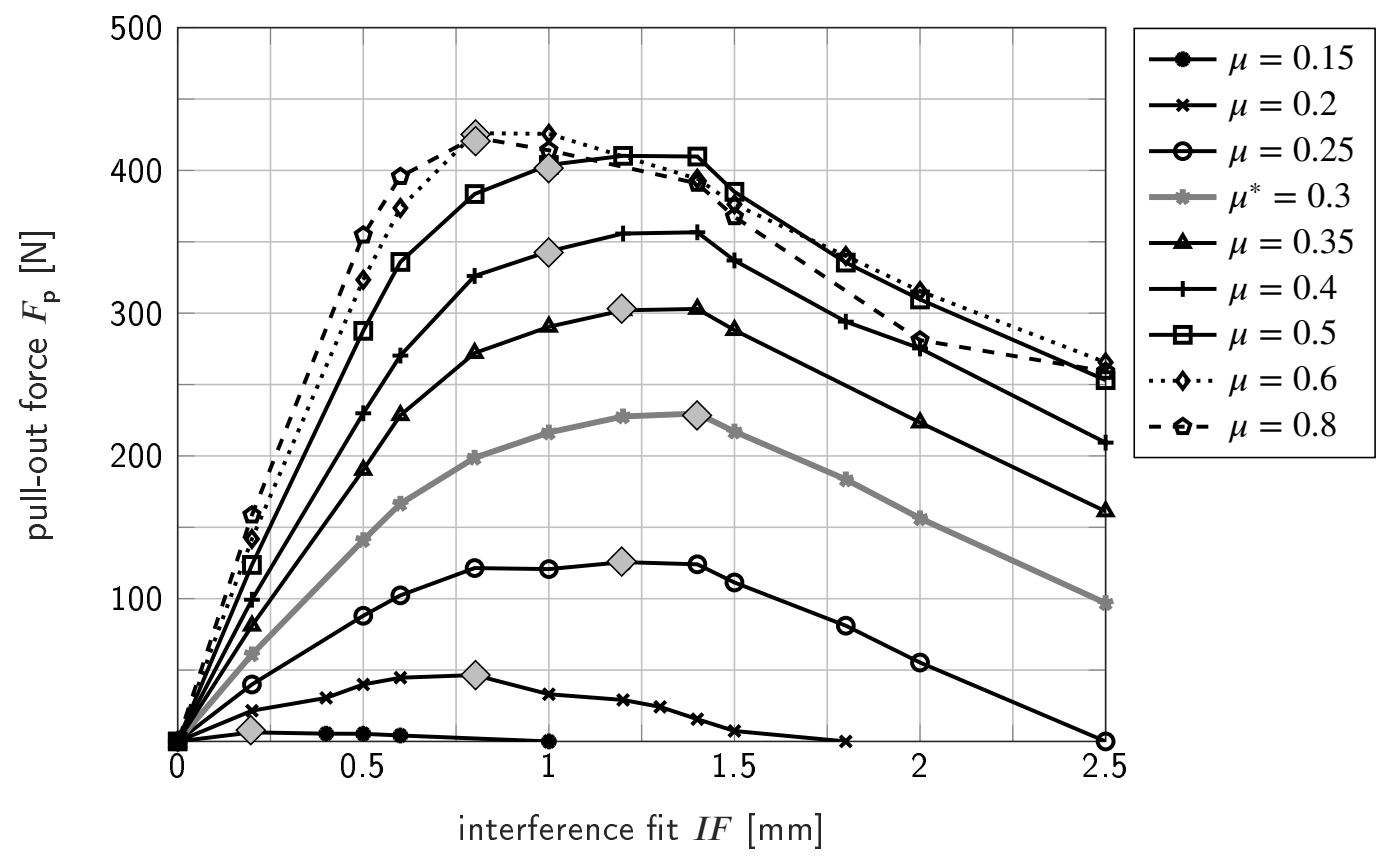

Figure 8: Variation of the pull-out force $F_{\mathrm{p}}$ as a function of the interference fit $I F$ and the friction coefficient $\mu$, for constant Young's moduli $E_{\mathrm{c}}^{*}=18 \mathrm{GPa}, E_{\mathrm{t}}^{*}=0.2 \mathrm{GPa}$. The optimal interference fit $I F_{\text {opt }}$ for each constellation is marked with a diamond.

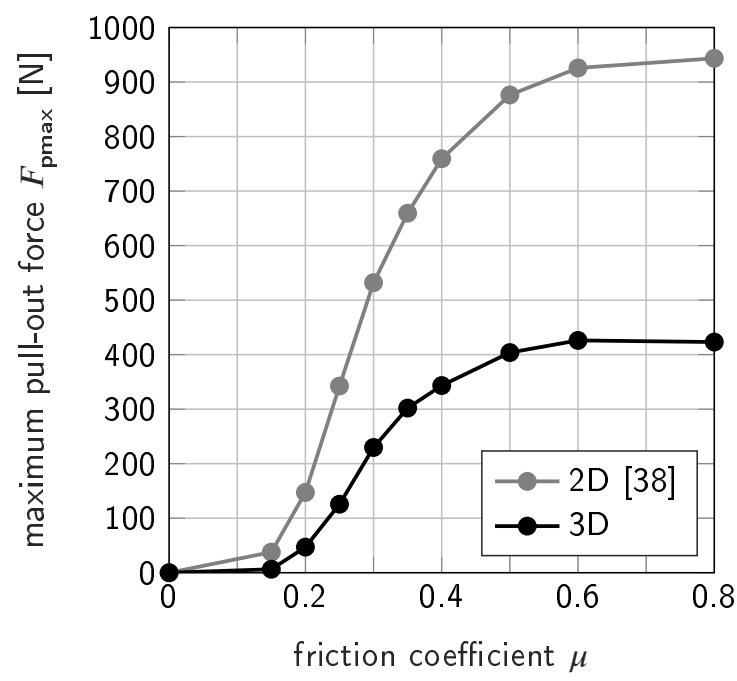

Figure 9: Variation of the maximal pull-out force $F_{\text {pmax }}$ obtained for the optimal interference fit $I F_{\text {opt }}$ as a function of the friction coefficient $\mu$ for constant Young's moduli $E_{\mathrm{c}}^{*}=18$ $\mathrm{GPa}, E_{\mathrm{t}}^{*}=0.2 \mathrm{GPa}$.

force monotonically increases as a function of $E_{\mathrm{t}}$. Since in our study and in e.g., Spears et al. (1999) [47], a $1 \mathrm{~mm}$ thick cortical layer is present, the difference in the magnitude of the pull-out force obtained with the 2D models [38, 47] and with the present 3D model might be explained by the differences in the geometry of the acetabulum, the boundary conditions, and the corresponding structural stiffness. Both considered 2D studies [38, 47] used axisymmetric, simplified models of the pelvis with 1) a lower surface fixed in all

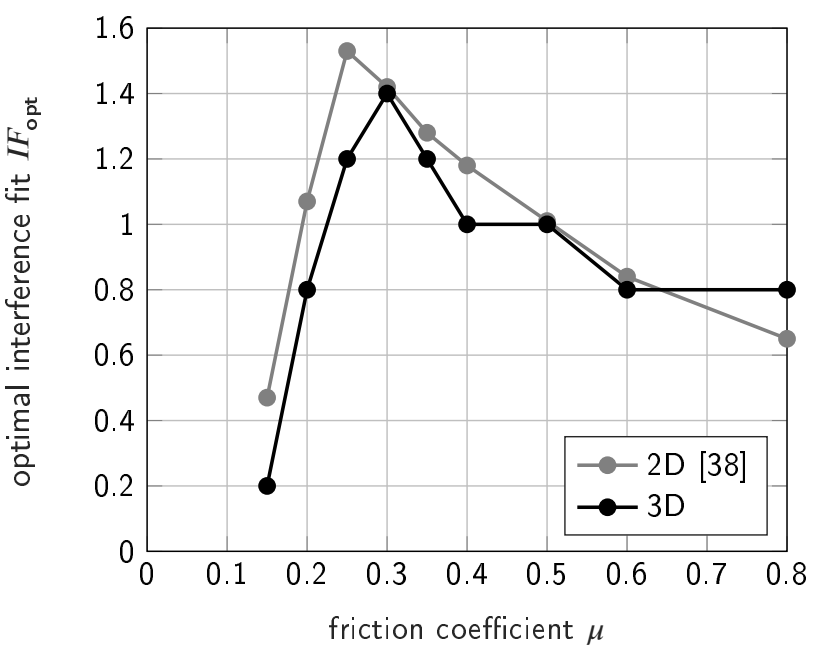

Figure 10: Variation of the optimal interference fit $I F_{0}$ as a function of the friction coefficient $\mu$. The results are shown for constant Young's moduli $E_{\mathrm{c}}^{*}=18 \mathrm{GPa}, E_{\mathrm{t}}^{*}=0.2 \mathrm{GPa}$.

directions and 2) a perfectly hemi-spherical cavity. In contrast, the present work considered a 3D hemi-pelvis model, which is fixed at the pubic symphysis and the iliac joint only. The cavity is not perfectly hemispherical since the acetabular wall is not present everywhere (see Figure 1B), similarly to what is obtained in the clinic. Moreover, the wall-thickness of the cavity rim is much lower and non-uninform in the 3D model, implying lower wall stiffness, and hence lower contact pressures and pull-out forces, as shown in Figure 2. In particular, for the 3D model 1) the linear response during the insertion phase is considerably shorter, 2) the reaction 
force rises more slowly during the insertion and the pullout phase, and 3) the pull-out force is much smaller than in the in the 2D model [38]. However, the present study shows that the 3D hemi-pelvis geometry does not influence the overall response of the pull-out force to different friction coefficients and interference fit when compared to a $2 \mathrm{D}$ axisymmetric setup [38] (see Figures 6A and 7A). Nonetheless, the real 3D geometry is shown to have a significant effect on the response of the pull-out force to trabecular bone stiffness (see Figure 4A). Therefore, when analyzing the implant stability in terms of the pull-out force and the polar gap, modeling a realistic pelvis geometry is necessary, which is consistent with previous studies studies [23].

\subsection{Polar gap}

While bone ingrowth has been shown for polar gaps up to $5 \mathrm{~mm}$ [32], the potential osteocyte jumping distance is deemed to be no greater than $1 \mathrm{~mm}[22,29]$. Several studies agree that the initial polar gap should not exceed 500 $\mu \mathrm{m}$, as higher gaps can promote the growth of weaker bone tissue $[32,41]$ or inhibit bone growth completely, and thus jeopardize long-term stability [22, 42]. Polar gaps observed in the clinic are usually referred to being "lower than $500 \mu \mathrm{m}$ and never higher than $1 \mathrm{~mm}$ " [42, 49]. In this study, high pull-out forces often coincide with large polar gaps (> 500 $\mu \mathrm{m})$ and thus, a balance between maximizing the pull-out force and minimizing the polar gap should be targeted.

As shown in Figures 4B-7B, the polar gap increases as a function of $E_{\mathrm{c}}, E_{\mathrm{t}}, \mu, I F$. While assuming the reference values for all other parameters, values of $E_{\mathrm{t}}$ higher than 0.4 $\mathrm{GPa}$ lead to polar gaps above $500 \mu \mathrm{m}$. Therefore, and as shown in Figures 6 and 7, to balance out the pull-out force and the size of the polar gap, high friction coefficients and a large interference fit should be avoided for patients with stiffer bone. Since low interfacial friction may destabilize the implant [21], a reduction of the interference fit might be favorable in such cases.

Polar gaps values are found to be between 0 and 2483 $\mu \mathrm{m}$ in this study, which is in agreement with the findings of other clinical [32, 42, 49], experimental [26, 29] and FE studies $[1,40,54]$. The polar gap is found to increase with larger interference fit [29], as well as with bone stiffness and friction coefficient. In our study, the best compromise between a high pull-out force and a polar gap $\leq 500 \mu \mathrm{m}$ for an interference fit of $1 \mathrm{~mm}$ is obtained for $\mu_{\mathrm{opt}}=0.5$ for $E_{\mathrm{t}}^{*}=0.2 \mathrm{GPa}$ and for $\mu_{\mathrm{opt}}=0.6$ for $E_{\mathrm{t}}=0.1 \mathrm{GPa}$. For $E_{\mathrm{t}}$ $=0.5 \mathrm{GPa}$, only friction coefficients $\mu \leq 0.2$ lead to values of polar gaps below $500 \mu \mathrm{m}$ but in this case, an insufficient pull-out force is obtained. Accepting a polar gap of up to 1 $\mathrm{mm}$ leads to $\mu=0.5$ as the optimal choice. In general, polar gaps predicted in this study are significantly lower than in comparable 2D axisymmetric studies [38, 47], which may be explained by the fact that 2D configurations are much stiffer compared to the 3D case (see above). Optimal friction coefficients are found to be higher in our study, compared to e.g., Spears et al. (1999) [47] ( $\mu=0.2-0.3)$, while Raffa et al. (2019) [38] found an optimum for $\mu=0.6$, as only the pull-out force was considered as a determinant of primary stability.

\subsection{Contact Stress}

The maximal value of the contact stresses on the cavity surface is found to be localized at the equatorial rim, which is in good agreement with previous works by several authors [1, 17, 21, 27, 47] who established that the contact between the ACI and the surrounding bone tissue mostly occurs around the equatorial rim. As shown in Figure 3, the maximum value of the contact pressure for the reference configuration is found to be around $10 \mathrm{MPa}$, which is in good agreement with similar studies $[38,52]$. The maximal contact stress observed in the optimal case for $E_{\mathrm{t}}$ is twice as high as in the reference case (data not shown), which corresponds to the increased pull-out force observed for the optimal case. However, excessive stresses can lead to bone damage during the implantation or to bone resorption later and thus should be monitored carefully.

\subsection{Optimal conditions for primary stability}

Several studies suggest that the interference fit is one of the most important factors in order to achieve adequate fixation $[10,28,47]$ and that increased under-reaming can compensate low bone stiffness [21, 40]. As low interfacial friction may destabilize the implant [21] and thus has to be avoided, an adequate interference fit according to the bone quality must be chosen instead.

While it was shown that higher interference fit values are required for softer bone $\left(E_{\mathrm{t}}=0.1 \mathrm{GPa}\right)$ to achieve similar pull-out forces as in the reference case $\left(E_{\mathrm{t}}^{*}=0.2 \mathrm{GPa}\right)$, common interference fit values (between 1 and $2 \mathrm{~mm}$ ) lead to excessive gaps for stiffer bone $\left(E_{\mathrm{t}}=0.5 \mathrm{GPa}\right)$ and thus should be avoided. However, our findings suggest that the interference fit should not be increased to more than around $1.3 \mathrm{~mm}$, because it leads to a concomitant decrease of the pull-out force and increase of the polar gap. For both, the 2D axisymmetric and 3D cases, an optimal interference fit of $I F=1.4$ for $E_{\mathrm{t}}^{*}=0.2 \mathrm{GPa}$ and $\mu^{*}=0.3$ is found, when only considering maximizing the pull-out force, which is in agreement with other studies [26, 28].

Previous studies confirm our findings concerning the existence of an optimal primary stability condition linking pressfitting and friction [26, 27, 38, 47]. In our study an interference fit of around $1.3 \mathrm{~mm}$ is found to be optimal in terms of maximal pull-out force for friction coefficients ranging between $\mu=0.25-0.5$ and $E_{\mathrm{t}}^{*}=0.2 \mathrm{GPa}$, while a mean interference fit of $1.1 \mathrm{~mm}$ is found optimal when considering a polar gap less than $500 \mu \mathrm{m}$ as well, similar to comparable studies [26, 27, 38, 47]. However, there are also studies that suggest a larger interference fit of $I F=2-3 \mathrm{~mm}$ [27].

The optimal values of $I F$ and $\mu$ in terms of maximal pull-out force and minimal polar gap for the different values of trabecular bone stiffness are listed in Table 2. As the optimal friction coefficient is higher than in the reference case, while the interference fit is lower, the determined optimal cases suggest that a higher friction coefficient (up to $\mu=$ 
0.6) enhances primary stability more than an increased interference fit. While being optimal in terms of the polar gap and the pull-out force, the determined optimal cases exhibit a higher stress in the equatorial rim of the cavity compared to the reference configuration. Excessive stresses in the cavity can become critical because they can cause bone damage during the insertion and bone resorption during the healing phase [45]. Therefore, future analyses of optimal primary stability should also include and classify the hoop stress in the cavity.

\subsection{Limitations}

This study has several limitations. First, only a single pelvis geometry of unknown sex and age was considered. Moreover, in the context of hip replacements, pathological degeneration of the bone structure and damage often need to be considered. Due to the numerous parameters considered and to the considerable computation time, different patient geometries and implantation angles could not be taken into account here and the impact of changes of the anatomy should be considered in future work, since bone geometry and stiffness have an effect on implant stability [8]. Several recent studies analyzed the influence of different patient geometries and bone stiffness [8,37], as well as the influence of different implantation angles [13, 21]. Second, all materials were assumed to be homogeneous and isotropic as is mostly done in the literature $[7,8,21]$. This assumption allows to assess the influence of a small number of parameters in a controlled manner.

Furthermore, the observed stress in the contact area was below the yield stress of human bone [3, 31]. Therefore, it may be assumed that no bone damage took place for the simulated insertion and pull-out tests, allowing to consider a hypoelastic material model as a valid simplification. While trabecular bone damage may occur during the insertion process, an experimental study showed that bone damage has no impact on the pull-out force [4]. As our study focuses on initial stability in terms of the pull-out force, bone damage was neglected as well.

Several studies consider the inhomogeneous $[8,40]$, anisotropic [33], and plastic [46] nature of bone tissues, by using data obtained from CT scans or experiments with sawbones. Other studies have also considered elasto-plastic effects [21, 27, 36], debonding [20], and damage of the interface $[36,39]$. While some studies point out the need to explicitly model the nonuniform thickness of the cortical layer $[8,19]$, realistic results have also been achieved with a uniform cortical thickness around 0.9-1.5 mm [2, 37, 51]. Due to the simulated reaming in our study, there is no cortical bone remaining in the contact area for any interference fit value (see Figure 1B) so the ACI only comes into contact with trabecular bone. As shown in Figure 5,the influence of the cortical bone stiffness on the pull-out force and on the polar gap in our model is relatively small compared to other studied parameters. Thus, we assumed that the effect of the cortical bone thickness was negligible compared to the effect of other parameter, which is in agreement with the literature $[2,37,51]$. However, when considering the stress and deformation distributions over the whole pelvis, the cortical bone thickness must be taken into account. Third, as this study focuses on primary stability during surgery, only a pull-out test and no cyclic loading (e.g., walking) was simulated. Thus, the influence of muscle tissue and ligaments on the deformation behavior and load response was neglected, which is in agreement with what is done in the literature [8, 14]. However, it has been shown that muscles and ligaments have to be taken into account when analyzing the stress distribution inside the acetabulum [43]. To draw comparisons to a previous study [38], a normal pull-out test was chosen, although lever-out tests have also been used in the literature [28]. Fourth, a quasi-static configuration was considered, and all dynamic aspects were neglected, similarly to what was done in some comparable works [27, 38, 47]. Note that a previous study precisely focuses on the insertion process of an ACI by considering dynamic modeling [30], which is important when modeling the insertion by hammer impacts. However, using dynamic modeling would not modify the pull-out test performed in this study. Fifth, only the pull-out force and the polar gap have been chosen as determinants of the ACI primary stability and the detailed stress distribution at the BII for each parameter set was not studied specifically. The stress distribution especially at the equatorial rim of the cavity should be analyzed and classified as well, as is done in other works [21, 40]. Micromotion at the BII, as well as contact area are other determinants of initial stability used in the literature [21, 40]. Furthermore, the polar gap was obtained at a given insertion force, which is the same for all cases considered in this work. However, when the insertion force is removed once the implant is inserted, the polar gap can increase due to relaxation [30]. Although this situation is clinically relevant, it is out of the scope of this work but will be considered in future studies. In addition, a mathematical relationship for the trends of the relationship between bone stiffness, interference fit, and friction coefficient (shown in Figures 6A, 7A, and 9) should be established. Including the aforementioned factors in future works could provide a more complete picture on implant primary stability and facilitate the choice of an implant configuration for a specific patient and provide a basis for modeling osseointegration.

Eventually, the proposed model should be validated experimentally as is partially done in the 2D case [38]. However, drawing comparisons to e.g., clinical studies is difficult, due to all the assumptions described above. Moreover, it remains difficult to measure the actual friction coefficient of the BII [12], as well as the elastic bone modulus (bone being heterogeneous, viscoelastic, and anisotropic) experimentally. Furthermore, as the hip cavity is reamed by hand, it is difficult to achieve a perfectly hemispherical cavity and it has been shown that the actual interference fit differs from what is determined by the implant and the last reamer size used $[23,28]$. 


\section{Conclusion}

This study investigates the dependence of the ACI primary stability on biomechanical parameters including the interference fit of the implant, the friction coefficient of the bone-implant interface, and bone tissue elasticity. An insertion and subsequent removal test allow to determine the polar gap and the pull-out force as a function of the four aforementioned parameters. Assuming a realistic pelvis geometry has a significant effect on the influence of $\mu, I F, E_{\mathrm{t}}$, and $E_{\mathrm{c}}$ on the ACI primary stability. All studied parameters $E_{\mathrm{t}}, E_{\mathrm{c}}, I F$, and $\mu$ significantly influence the ACI primary stability. Quadratic regression analyses were used for the dependence of the pull-out force on the different parameters and linear regression analyses were performed to analyze the dependence of the polar gap on the same parameters. All $\mathrm{p}$-values were $\mathrm{p}<0.01$ (where $\mathrm{p}$ is the probability that the null hypothesis is true). An optimal combination of $\mu$ and $I F$ was determined. For $E_{\mathrm{t}}=0.1 \mathrm{GPa}$, the optimal configuration corresponds to $I F=0.8 \mathrm{~mm}$ and $\mu=$ 0.6 , while for $E_{\mathrm{t}}=0.2 \mathrm{GPa}$, it corresponds to $I F=0.8 \mathrm{~mm}$ and $\mu=0.6$ and for $E_{\mathrm{t}}=0.5 \mathrm{GPa}$, it is $I F=0.8 \mathrm{~mm}$ and $\mu=0.5$. The strong correlation between the aforementioned parameters may therefore require particular attention of implant manufacturers and of surgeons in order to maximize the ACI primary stability. The numerical results are found to be consistent with previous experimental and numerical studies and can help surgeons select the optimal interference fit in a patient-specific manner, based on the patient's bone quality and the chosen implant. The results also show that increasing $I F$ above $1.4 \mathrm{~mm}$ and $\mu$ above 0.6 has no benefit on ACI primary stability, which can aid in ACI implant conception and selection of appropriate surface treatments. In addition, this study provides detailed knowledge of the local contact state and the influence of implant- and patientspecific parameters and hence, is an important step towards modeling and understanding osseointegration. The results presented in this work can be used as a basis for modeling long-term stability, e.g., for stress-, strain- or micromotion-dependent osseointegration models and the subsequent debonding of osseointegrated implants [20,39]. This model can also be applied to hip geometries with osseous defects to provide suggestions for ensuring primary stability for these challenging and clinically relevant cases. However, the proposed in silico model needs to be improved to better match the clinical conditions, e.g., by simulating leverout tests or including different hip geometries and inhomogeneous bone stiffness. As the problem is characterized by multi-parameter optimization, a rigorous determination of optimal parameter combinations requires corresponding optimization algorithms, which should be considered in future works.

\section{Acknowledgments}

This work has received funding from the European Research Council (ERC) under the European Union's Horizon 2020 research and innovation program (Grant Agreement
No 682001, Project ERC Consolidator Grant 2015 BoneImplant) and was supported by the Jülich Aachen Research Alliance Center for Simulation and Data Science (JARA-CSD) School for Simulation and Data Science (SSD). The authors would like to thank Lucie Flammand and Adrien Ledoux for help in obtaining numerical results.

\section{References}

[1] Amirouche, F., Solitro, G., Broviak, S., Gonzalez, M., Goldstein, W., Barmada, R., 2014. Factors influencing initial cup stability in total hip arthroplasty. Clinical Biomechanics 29, 1177-1185.

[2] Anderson, A.E., Peters, C.L., Tuttle, B.D., Weiss, J.A., 2005. Subjectspecific finite element model of the pelvis: development, validation and sensitivity studies. Journal of Biomechanical Engineering 127, 364-373.

[3] Bayraktar, H.H., Morgan, E.F., Niebur, G.L., Morris, G.E., Wong, E.K., Keaveny, T.M., 2004. Comparison of the elastic and yield properties of human femoral trabecular and cortical bone tissue. Journal of biomechanics 37, 27-35.

[4] Bishop, N.E., Höhn, J.C., Rothstock, S., Damm, N.B., Morlock, M.M., 2014. The influence of bone damage on press-fit mechanics. Journal of biomechanics 47, 1472-1478.

[5] Brånemark, P.I., 1977. Osseointegrated implants in the treatment of the edentulous jaw. experience from a 10-year period. Scand. J. Plast. Reconstr. Surg. Suppl. 16.

[6] Cignoni, P., Callieri, M., Corsini, M., Dellepiane, M., Ganovelli, F., Ranzuglia, G., 2008. Meshlab: an open-source mesh processing tool., in: Eurographics Italian chapter conference, Salerno, Italy. pp. 129136.

[7] Cilingir, A.C., Ucar, V., Kazan, R., 2007. Three-dimensional anatomic finite element modelling of hemi-arthroplasty of human hip joint. Trends Biomater Artif Organs 21, 63-72.

[8] Clarke, S., Phillips, A., Bull, A., 2013. Evaluating a suitable level of model complexity for finite element analysis of the intact acetabulum. Computer methods in biomechanics and biomedical engineering 16 , 717-724.

[9] Cochran, D., Schenk, R., Lussi, A., Higginbottom, F., Buser, D., 1998. Bone response to unloaded and loaded titanium implants with a sandblasted and acid-etched surface: A histometric study in the canine mandible. Journal of Biomedical Materials Research: An Official Journal of The Society for Biomaterials, The Japanese Society for Biomaterials, and the Australian Society for Biomaterials 40, 1-11.

[10] Curtis, M., Jinnah, R., Wilson, V., Hungerford, D., 1992. The initial stability of uncemented acetabular components. The Journal of bone and joint surgery. British volume 74, 372-376.

[11] Dammak, M., Shirazi-Adl, A., Schwartz Jr, M., Gustavson, L., 1997. Friction properties at the bone-metal interface: Comparison of four different porous metal surfaces. Journal of Biomedical Materials Research 35, 329-336.

[12] Gao, X., Fraulob, M., Haïat, G., 2019. Biomechanical behaviours of the bone-implant interface: a review. Journal of the Royal Society Interface 16, 20190259.

[13] Goldman, A.H., Armstrong, L.C., Owen, J.R., Wayne, J.S., Jiranek, W.A., 2016. Does increased coefficient of friction of highly porous metal increase initial stability at the acetabular interface? The Journal of arthroplasty 31, 721-726.

[14] Hao, Z., Wan, C., Gao, X., Ji, T., 2011. The effect of boundary condition on the biomechanics of a human pelvic joint under an axial compressive load: a three-dimensional finite element model. Journal of Biomechanical Engineering 133, 101006.

[15] hemi-pelvis STL file, . Lincoln 3D scans. https: //www. lincoln3dscans. co. uk/lincoln-3d-scans/2018/7/3/ human-pelvis-bone. Accessed: 11.06.2019.

[16] Henyš, P., Čapek, L., 2017. Material model of pelvic bone based on modal analysis: a study on the composite bone. Biomechanics and modeling in mechanobiology 16, 363-373. 
[17] Hothi, H., Busfield, J., Shelton, J., 2011. Explicit finite element modelling of the impaction of metal press-fit acetabular components. Proceedings of the Institution of Mechanical Engineers, Part H: Journal of Engineering in Medicine 225, 303-314.

[18] Hsu, J.T., Chang, C.H., Huang, H.L., Zobitz, M.E., Chen, W.P., Lai, K.A., An, K.N., 2007. The number of screws, bone quality, and friction coefficient affect acetabular cup stability. Medical Engineering \& Physics 29, 1089-1095.

[19] Hsu, J.T., Lai, K.A., Chen, Q., Zobitz, M.E., Huang, H.L., An, K.N., Chang, C.H., 2006. The relation between micromotion and screw fixation in acetabular cup. Computer Methods and Programs in Biomedicine 84, 34-41.

[20] Immel, K., Duong, T.X., Nguyen, V.H., Haiat, G., Sauer, R.A., 2020. A modified coulomb's law for the tangential debonding of osseointegrated implants. Biomechanics and modeling in mechanobiology, $1-18$.

[21] Janssen, D., Zwartelé, R.E., Doets, H.C., Verdonschot, N., 2010. Computational assessment of press-fit acetabular implant fixation: the effect of implant design, interference fit, bone quality, and frictional properties. Proceedings of the Institution of Mechanical Engineers, Part H: Journal of Engineering in Medicine 224, 67-75.

[22] Jasty, M., Summer, R., Galante, J.O., Bragdon, C.R., Cargill, E.A., Harris, W.H., 1988. Bone ingrowth into porous-surfaced harris/galante prosthesis acetabular components retrived from human patients, in: Presented at the 55th Annual Meeting of the American Academy of Orthopaedic Surgeons. Atlanta, Georgia.

[23] Kim, Y., Brown, T., Pedersen, D., Callaghan, J., 1995. Reamed surface topography and component seating in press-fit cementless acetabular fixation. The Journal of arthroplasty 10, S14-S21.

[24] Kremers, H.M., Howard, J.L., Loechler, Y., Schleck, C.D., Harmsen, W.S., Berry, D.J., Cabanela, M.E., Hanssen, A.D., Pagnano, M.W., Trousdale, R.T., et al., 2012. Comparative long-term survivorship of uncemented acetabular components in revision total hip arthroplasty. JBJS 94, e82.

[25] Kurtz, S., Ong, K., Lau, E., Mowat, F., Halpern, M., 2007. Projections of primary and revision hip and knee arthroplasty in the united states from 2005 to 2030. JBJS 89, 780-785.

[26] Kwong, L.M., O'Connor, D.O., Sedlacek, R.C., Krushell, R.J., Maloney, W.J., Harris, W.H., 1994. A quantitative in vitro assessment of fit and screw fixation on the stability of a cementless hemispherical acetabular component. The Journal of Arthroplasty 9, 163-170.

[27] Le Cann, S., Galland, A., Rosa, B., Le Corroller, T., Pithioux, M., Argenson, J.N., Chabrand, P., Parratte, S., 2014. Does surface roughness influence the primary stability of acetabular cups? a numerical and experimental biomechanical evaluation. Medical Engineering \& Physics 36, 1185-1190.

[28] Macdonald, W., Carlsson, L., Charnley, G., Jacobsson, C., 1999. Press-fit acetabular cup fixation: principles and testing. Proceedings of the Institution of Mechanical Engineers, Part H: Journal of Engineering in Medicine 213, 33-39.

[29] MacKenzie, J.R., Callaghan, J.J., Pedersen, D.R., Brown, T.D., 1994. Areas of contact and extent of gaps with implantation of oversized acetabular components in total hip arthroplasty. Clinical orthopaedics and related research , 127-136.

[30] Michel, A., Nguyen, V.H., Bosc, R., Vayron, R., Hernigou, P., Naili, S., Haiat, G., 2017. Finite element model of the impaction of a pressfitted acetabular cup. Medical \& Biological Engineering \& Computing 55, 781-791.

[31] Morgan, E.F., Unnikrisnan, G.U., Hussein, A.I., 2018. Bone mechanical properties in healthy and diseased states. Annual review of biomedical engineering 20, 119-143.

[32] Mukherjee, K., Gupta, S., 2014. Simulation of tissue differentiation around acetabular cups: the effects of implant-bone relative displacement and polar gap. Adv. Biomech. Appl 1, 95-109.

[33] Nguyen, V.H., Rosi, G., Naili, S., Michel, A., Raffa, M.L., Bosc, R., Meningaud, J.P., Chappard, C., Takano, N., Haiat, G., 2017. Influence of anisotropic bone properties on the biomechanical behavior of the acetabular cup implant: a multiscale finite element study.
Computer Methods in Biomechanics and Biomedical Engineering 20, $1312-1325$.

[34] Novitskaya, E., Chen, P., Hamed, E., Jun, L., Lubarda, V.A., Jasiuk, I., McKittrick, J., 2011. Recent advances on the measurement and calculation of the elastic moduli of cortical and trabecular bone: a review. Theoretical and Applied Mechanics 38, 209-297.

[35] Olory, B., Havet, E., Gabrion, A., Vernois, J., Mertl, P., 2004. Comparative in vitro assessment of the primary stability of cementless press-fit acetabular cups. Acta Orthopædica Belgica 70, 31-37.

[36] Ovesy, M., Aeschlimann, M., Zysset, P.K., 2020. Explicit finite element analysis can predict the mechanical response of conical implant press-fit in homogenized trabecular bone. Journal of Biomechanics, 109844.

[37] Phillips, A., Pankaj, P., Howie, C., Usmani, A., Simpson, A., 2007. Finite element modelling of the pelvis: inclusion of muscular and ligamentous boundary conditions. Medical Engineering \& Physics 29, 739-748.

[38] Raffa, M.L., Nguyen, V.H., Tabor, E., Immel, K., Housset, V., Flouzat-Lachaniette, C.H., Haiat, G., 2019. Dependence of the primary stability of cementless acetabular cup implants on the biomechanical environment. Proceedings of the Institution of Mechanical Engineers, Part H: Journal of Engineering in Medicine 233, 12371249.

[39] Rittel, D., Dorogoy, A., Shemtov-Yona, K., 2018. Modeling the effect of osseointegration on dental implant pullout and torque removal tests. Clinical Implant Dentistry and Related Research 20, 683-691.

[40] Rourke, D.O., Taylor, M., 2020. Patient and surgical variability in the primary stability of cementless acetabular cups: a finite element study. Journal of Orthopaedic Research .

[41] Sandborn, P.M., Cook, S.D., Spires, W.P., Kester, M.A., 1988. Tissue response to porous-coated implants lacking initial bone apposition. The Journal of arthroplasty 3, 337-346.

[42] Schmalzried, T.P., Kwong, L.M., Jasty, M., Sedlacek, R.C., Haire, T.C., O'Connor, D.O., Bragdon, C.R., Kabo, J.M., Malcolm, A.J., Harris, W.H., 1992. The mechanism of loosening of cemented acetabular components in total hip arthroplasty. analysis of specimens retrieved at autopsy. Clinical orthopaedics and related research , 6078.

[43] Shirazi-Adl, A., Dammak, M., Paiement, G., 1993. Experimental determination of friction characteristics at the trabecular bone/porouscoated metal interface in cementless implants. Journal of Biomedical Materials Research 27, 167-175.

[44] Søballe, K., Hansen, E.S., B.-Rasmussen, H., Jørgensen, P.H., Bünger, C., 1992. Tissue ingrowth into titanium and hydroxyapatitecoated implants during stable and unstable mechanical conditions. Journal of Orthopaedic Research 10, 285-299.

[45] Sotto-Maior, B.S., Rocha, E.P., Almeida, E.O.d., Freitas-Júnior, A.C., Anchieta, R.B., Cury, A.A.D.B., 2010. Influence of high insertion torque on implant placement: an anisotropic bone stress analysis. Brazilian Dental Journal 21, 508-514.

[46] Souffrant, R., Zietz, C., Fritsche, A., Kluess, D., Mittelmeier, W., Bader, R., 2012. Advanced material modelling in numerical simulation of primary acetabular press-fit cup stability. Computer Methods in Biomechanics and Biomedical Engineering 15, 787-793.

[47] Spears, I.R., Morlock, M.M., Pfleiderer, M., Schneider, E., Hille, E., 1999. The influence of friction and interference on the seating of a hemispherical press-fit cup: a finite element investigation. Journal of Biomechanics 32, 1183-1189.

[48] Toossi, N., Adeli, B., Timperley, A.J., Haddad, F.S., Maltenfort, M., Parvizi, J., 2013. Acetabular components in total hip arthroplasty: is there evidence that cementless fixation is better? JBJS 95, 168-174.

[49] Udomkiat, P., Dorr, L.D., Wan, Z., 2002. Cementless hemispheric porous-coated sockets implanted with press-fit technique without screws: average ten-year follow-up. JBJS 84, 1195-1200.

[50] Ulrich, S.D., Seyler, T.M., Bennett, D., Delanois, R.E., Saleh, K.J., Thongtrangan, I., Kuskowski, M., Cheng, E.Y., Sharkey, P.F., Parvizi, J., et al., 2008. Total hip arthroplasties: what are the reasons for revision? International Orthopaedics 32, 597-604. 
[51] Watson, P.J., Dostanpor, A., Fagan, M.J., Dobson, C.A., 2017. The effect of boundary constraints on finite element modelling of the human pelvis. Medical Engineering \& Physics 43, 48-57.

[52] Widmer, K.H., Zurfluh, B., Morscher, E., 2002. Load transfer and fixation mode of press-fit acetabular sockets. The Journal of arthroplasty 17, 926-935.

[53] Wriggers, P., 2006. Computational contact mechanics, springerverlag. Berlin, Heidelberg

[54] Yew, A., Jin, Z., Donn, A., Morlock, M., Isaac, G., 2006. Deformation of press-fitted metallic resurfacing cups. part 2: finite element simulation. Proceedings of the Institution of Mechanical Engineers, Part H: Journal of Engineering in Medicine 220, 311-319. 